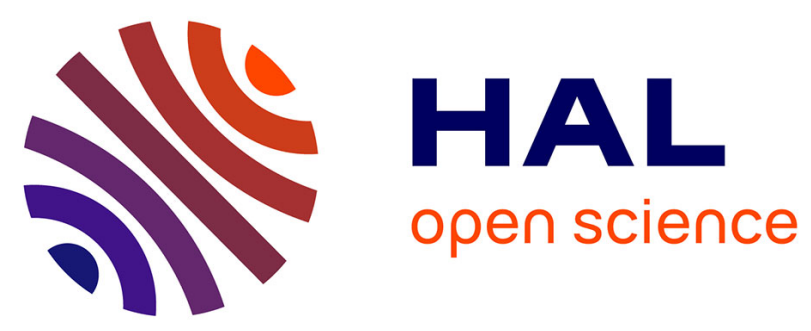

\title{
ULTRASONIC LIGHT DIFFRACTION FOR THE CASE OF THE INTENSE LASER BEAM GENERATING A 3rd HARMONIC IN LIQUIDS
}

J. Jozefowska, M. Kosmol, A. Sliwinski

\section{- To cite this version:}

J. Jozefowska, M. Kosmol, A. Sliwinski. ULTRASONIC LIGHT DIFFRACTION FOR THE CASE OF THE INTENSE LASER BEAM GENERATING A 3rd HARMONIC IN LIQUIDS. Journal de Physique Colloques, 1972, 33 (C6), pp.C6-239-C6-244. 10.1051/jphyscol:1972652 . jpa-00215170

HAL Id: jpa-00215170

https://hal.science/jpa-00215170

Submitted on 1 Jan 1972

HAL is a multi-disciplinary open access archive for the deposit and dissemination of scientific research documents, whether they are published or not. The documents may come from teaching and research institutions in France or abroad, or from public or private research centers.
L'archive ouverte pluridisciplinaire HAL, est destinée au dépôt et à la diffusion de documents scientifiques de niveau recherche, publiés ou non, émanant des établissements d'enseignement et de recherche français ou étrangers, des laboratoires publics ou privés. 


\title{
ULTRASONIC LIGHT DIFFRACTION FOR THE CASE OF THE INTENSE LASER BEAM GENERATING A 3rd HARMONIC IN LIQUIDS
}

\author{
J. JOZEFOWSKA $\left({ }^{*}\right)$, M. KOSMOL and A. SLIWINSKI $\left({ }^{* *}\right)$
}

(*) Physics Laboratory, College of engineering, Bydgoszcz, Olszewskiego 20, Poland (**) Institute of Physics, University of Gdaǹsk, Gdaǹsk, Sobieskiego 18, Poland

Institut of Physics, University of Gdaǹsk, Gdaǹsk, Poland.

\begin{abstract}
Résumé. - Un calcul de la diffraction par une onde ultrasonore de la lumière intense émise par un laser est exposé dans le cas du sulfure de carbone, du tétrachlorure de carbone et du benzène.

Des essais ont été effectués pour trouver les conditions expérimentales qui confirment les prévisions théoriques.
\end{abstract}

\begin{abstract}
Some calculations of the intensity distributions of intense laser light diffracted on an ultrasonic wave were made for carbon disulfide, carbon tetrachloride and benzene.

Attempts have been made to find some proper conditions for the experiment to prove theoretical predictions.
\end{abstract}

1. Introduction. - Forty years have been passing since R. Lucas and P. Biquard [1], independently of P. Debye and F. W. Sears [2] carried out their experiments on ultrasonic light diffraction and proved the previous theoretical Brillouin's prediction [3]. These experiments became a starting point for many later works on this subect and discovered wide possibilities of new methods in ultrasonic research [1], [19].

When laser light of high intensity and conveying higher harmonics beside the fundamental frequency became available and diffracted by ultrasound, the new problem arose of whether the intensity distribution in diffraction patterns would be the same as in the case of low intensity usual light. In [20] the approximate formulas were derived for calculating the intensity distribution of light diffracted by ultrasound in the case of interaction with a strong laser beam. These formulas consider the effect up to the \pm 2 nd orders of diffraction for isotropic medium (in this case of laser beam propagation the 3rd harmonic of light is generated). The formulas of [20] were next changed slightly [21] assuming the other boundary conditions than previously.

In this paper we discuss and compare these two kinds of boundary conditions again and we present some further results of the numerical calculations of intensity distribution of diffraction patterns for the following liquids : nitrobenzene, carbon disulfide, carbon tetrachloride and benzene (results for nitrobenzene and carbon disulfide were already presented in [20] and [21]). They are liquids for which a relatively high effect of non linear change in refractive index was stated [22] and one can expect that the influence of it on the diffraction pattern of laser light due by ultrasound will be strong enough to measure it.

2. Incidence boundary conditions. - We assume a very intense monochromatic and coherent laser beam to traverse the isotropic medium in $z$ direction. Let the ultrasonic wave propagates in $x$ direction. We shall discuss two cases: a) Figure $1 a$ [20] and b) Figure $1 b$ [21]. In the case $a$ ) the laser beam enters the liquid at the plane $z=-l_{1}$, in the case, $b$ ) at $z=0$.
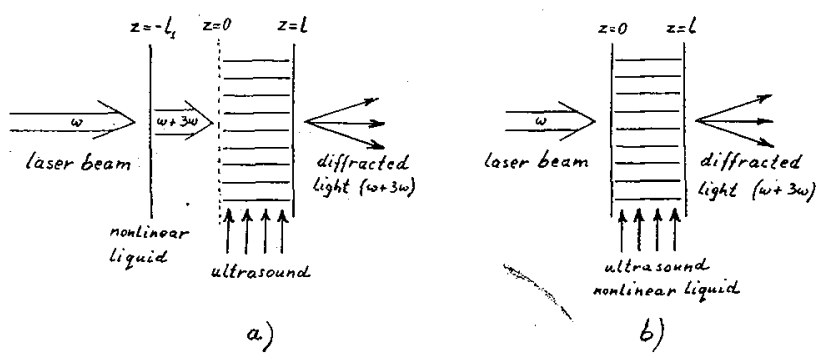

FrG. 1.

2.1 CASE $a$ ). - Starting from $z=-l_{1}$ the light sinusoidal wave is gradually deformed and as the result of this process the 3rd harmonic is generated. At the plane $z=0$ (Fig. 1a) the laser beam enters the ultrasonic wave traversing perpendicularly and they interact each other along the distance $l$. We assume that $l_{1}+l<l_{\mathrm{s}}$, where $l_{\mathrm{s}}$ is the coherence length for the 3rd harmonic. At $z=0$ the light beam consists of two components : the fundamental of frequency $\omega$ and the 3 rd harmonic of frequency $3 \omega$. The ratio of amplitudes of the harmonic to the fundamental depends on the distance $l_{1}$ and it is exactly deter- 
mined at the plane $z=0$. Let us assume that it is equal to

$$
r=\frac{\Phi_{0}^{3}(3 \omega, 0)}{\Phi_{0}^{0}(\omega, 0)},
$$

where $\Phi_{0}^{0}(\omega, 0)$ amplitude of the fundamental in liquid at $z=0, \Phi_{0}^{3}(3 \omega, 0)$ amplitude of the harmonic in liquid at $z=0$.

If $E\left(\omega-l_{1}\right)$ is the amplitude of the laser beam at the incidence plane from the air to the liquid, then we have the conditions :

$$
E\left(\omega,-l_{1}\right)=\Phi_{0}^{0}\left(\omega,-l_{1}\right) \text { for } z=-l_{1}
$$

and

$$
\left[\Phi_{0}^{3}(3 \omega, 0)\right]^{2}+\left[\Phi_{0}^{0}(\omega, 0)\right]^{2}=E^{2}\left(\omega,-l_{1}\right)
$$

for

$$
z=0
$$

whence using (1) we have

$$
\left[\Phi_{0}^{0}(\omega, 0)\right]^{2}=\frac{E^{2}\left(\omega,-l_{1}\right)}{1+r^{2}},
$$

it is the intensity (a square of amplitude) of the fundamental component of the laser beam at the plane $z=0$ where it enters the layer of interaction with ultrasonics.

For a very thin ultrasonic layer $\left(l \ll l_{1}\right)$ one can assume that the contribution from the generation of the 3rd harmonic within the layer is very small (comparing with the distance $l_{1}$ ) and in the first approximation can be not taken into account. Then the situation is like that described in [20] and the ultrasonic light diffraction process is treated as the superposition of two diffraction patterns of the two components i. e. fundamental and harmonic. The approximate calculated distribution of amplitudes of diffracted light beams at the output plane from the ultrasonic layer at $z=l$ (see [20]) is following :

$$
\begin{aligned}
& E(\omega, l, x, t)=\exp (2 \pi i v t) \sum_{k=-\infty}^{+\infty} \Phi_{k}^{0}(a) \times \\
& \quad \times \exp \left(-i \frac{n_{0}}{\delta n} a\right) \cdot \exp \left(2 \pi i k \frac{x}{\Lambda}\right) \cdot \exp (-2 \pi i N t) \\
& \quad+\exp [3(2 \pi i v t)] \sum_{k^{\prime}=-\infty}^{+\infty}\left[\Phi_{k^{\prime}}^{1}(3 a)+A_{k^{\prime}}(a)\right] \\
& \quad \times \exp \left(-i \frac{n_{0}}{\delta n} \cdot 3 a\right) \cdot \exp \left(2 \pi i k^{\prime} \frac{x}{\Lambda}\right) \\
& \quad \times \exp (-2 \pi i N t),
\end{aligned}
$$

for $k^{\prime}=3 k, k^{\prime}=0,1,2, \ldots$ where $v$ and $\lambda$ frequency and wavelength of light, respectively, $N$ and $A$ frequency and wavelength of ultrasound, $n_{0}$ light refractive index for undisturbed medium, $\delta n$ amplitude of variations in the index due to ultrasound propor- tional to the acoustic pressure of the wave, $x, z$ propagation direction of the ultrasonic wave and laser light wave, respectively, $t$ time. $\Phi_{k}^{0}(a)$ are Bessel functions of argument

$$
a=\frac{2 \pi \delta n l}{\lambda},
$$

$\Phi_{k^{\prime}}^{1}(3 a)$ are Bessel functions of the argument $3 a . A_{k^{\prime}}(a)$ complicated linear combinations of products of are these Bessel functions of different orders [20].

The first term in the formula (5) presenting the null approximation is identical with the distribution of Raman-Nath [5] but the second one determines the contribution which appears due to the presence of the 3rd harmonic in the light beam.

As the result of such a distribution of light and due to the interference the diffraction beams of different orders occur like it is shown in the figure 2.

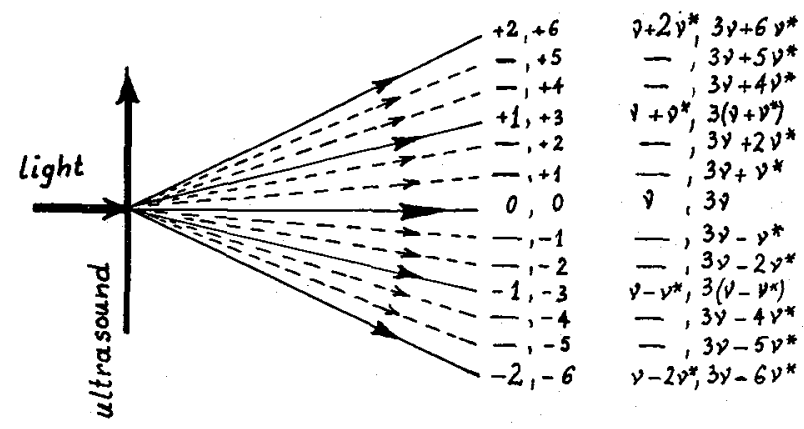

FIG. 2.

The continuous lines correspond to the diffraction of the fundamental component of laser beam (diffraction angles are determined by $\left.\operatorname{tg} \alpha_{k}=k \lambda / \Lambda\right)$ and the dotted lines to the $3 \mathrm{rd}$ harmonic (diffraction angles are determined by $\operatorname{tg} \alpha_{k^{\prime}}=k^{\prime} \lambda / 3 \Lambda$ ). Light in the particular fringes is frequency modulated due to the Doppler effect and the modulated frequencies are marked in the figure.

From the expr. (5) one can find the appropriate intensity distributions of light in different diffraction orders as the sum of the squared amplitudes. However, the exact calculation is not possible because the functions appearing in the solution of the second approximation are expressed by the third power of the series with the coefficients which are Bessel functions and they are also the solution of the null approximation [20]. Practically, it is possible to take into account only few terms of the series. In [20] it was assumed $k=0, \pm 1, \pm 2$ and from (5) the following approximate expressions were derived for the intensity distribution of light: for the 0-order (fundamental + harmonic), $k=0, k^{\prime}=0$ :

$$
\begin{gathered}
I_{0}=(1-r)^{2} \Phi_{0} \Phi^{*}+ \\
+E^{2} r^{2}\left[\Phi_{0}(3 a)+A_{0}(a)\right]\left[\Phi_{0}(3 a)+A_{0}(a)\right]^{*},
\end{gathered}
$$


for the 3 rd harmonic for the orders $k^{\prime}= \pm 1, \pm 2$ : $I_{ \pm 1}=E^{2} r^{2}\left[\Phi_{ \pm 1}(3 a)+A_{ \pm 1}(a)\right]\left[\Phi_{ \pm 1}(3 a)+A_{ \pm 1}(a)\right]^{*}$, $I_{ \pm 2}=E^{2} r^{2}\left[\Phi_{ \pm 2}(3 a)+A_{ \pm 2}(a)\right]\left[\Phi_{ \pm 2}(3 a)+A_{ \pm 2}(a)\right]^{*}$,

for the fundamental of the orders $k= \pm 1, \pm 2$ with the overlaped fringes of the harmonic for $k^{\prime}= \pm 3, \pm 6:$

$$
\begin{gathered}
I_{ \pm 1}=\left(1-r^{2}\right) \Phi_{-1} \Phi_{-1}^{*}+ \\
+E^{2} r^{2}\left[\Phi_{3}(3 a)+A_{3}(a)\right]\left[\Phi_{3}(3 a)+A_{3}(a)\right]^{*}, \\
I_{ \pm 2}=\left(1-r^{2}\right) \Phi_{-2} \Phi_{-2}^{*}+ \\
+E^{2} r^{2}\left[\Phi_{6}(3 a)+A_{6}(a)\right]\left[\Phi_{6}(3 a)+A_{6}(a)\right]^{*} .
\end{gathered}
$$

2.2 CASE $b$ ). - Now we assume that $r=0$ for $z=0($ Fig $1 b)$ which means that the laser beam does not contain the harmonic at the incidence plane of the ultrasonic layer but starts to be generated during the penetration across it. So, the second term in the expr. (5) will be changed in such a way that $\Phi_{k^{\prime}}^{1}(3 a)=0$ and $A_{k^{\prime}}(a)$ will be the only contribution to modify the amplitude distribution of light in the diffraction pattern. Thus we have

$$
\begin{aligned}
& E(\omega, l, x, t)=\exp (2 \pi v t) \sum_{k=-\infty}^{+\infty} \Phi_{k}^{0}(a) \exp \left(-i \frac{n_{0}}{\delta n} a\right) \times \\
& \quad \times \exp \left(2 \pi i k \frac{x}{\Lambda}\right) \cdot \exp (-2 \pi i N t) \\
& \quad+\exp (6 \pi i v t) \sum_{k^{\prime}=-\infty}^{+\infty} A_{k^{\prime}}(a) \exp \left(-i \frac{n_{0}}{\delta n} 3 a\right) \\
& \quad \times \exp \left(2 \pi i \frac{x}{\Lambda}\right) \cdot \exp (2 \pi i N t) .
\end{aligned}
$$

Calculations very similar like used in [20] lead to the following expressions for the intensity distribution in diffraction patterns of the harmonic

$$
\begin{gathered}
I_{0}=E^{2} A_{0}(a) A_{0}^{*}(a), \\
I_{ \pm 1}=E^{2} A_{ \pm 1}(a) A_{ \pm 1}^{*}(a), \\
I_{ \pm 2}=E^{2} A_{ \pm 2}(a) A_{*}^{ \pm 2}(a),
\end{gathered}
$$

and for the fundamental

$$
\begin{aligned}
& I_{ \pm 1}=E^{2} A_{3}(a) A_{3}^{*}(a), \\
& I_{ \pm 2}=E^{2} A_{6}(a) A_{6}^{*}(a) .
\end{aligned}
$$

Comparing this with the result for the case a) one can see that the general description of the phenomena is the same. There is no change in diffraction angles, frequency modulation and the shape of the diffraction patterns but the light intensity distribution is changed slightly.

(*) Conjugate complex.
3. Estimation of variations in refractive index due to the generation of the 3rd harmonic. - For the dielectric isotropic media the nonlinear optical properties can be described by the following relation between the electric field of the light wave $E$ and the polarization $P$ induced by it in the medium [25] :

$$
P=\alpha E+\beta E^{3}+\cdots
$$

$\alpha$ linear susceptibility, $\beta$ third order susceptibility (for the isotropic media and in the case of the crystals with the center of symmetry the term proportional to $E^{2}$ vanishes).

From the relation (9) it is possible to find the variation [23] of refractive index due to the nonlinear polarization. In general we have for the electric induction $D$ in the medium

$$
D=D_{0}+P
$$

where $D_{0}=\varepsilon_{0} E$ electric induction in vacuum, $\varepsilon_{0}$ electric permeability in vacuum. From the definition one can write

$$
\varepsilon_{E} \varepsilon_{0}=\frac{\mathrm{d} D}{\mathrm{~d} E}
$$

where $\varepsilon_{0}$ electric permeability which is the function of electric field $E$. From (11) basing on (10) and (9) we can derive [23]:

$$
n_{E}=n+\gamma E^{2} \quad \text { or } \quad \Delta n=n_{E}-n=\gamma E^{2}
$$

where

$$
\gamma=\frac{3 \beta}{2 \varepsilon_{0} n}, \quad n=\sqrt{\varepsilon}, \quad n_{E}=\sqrt{\varepsilon_{E}} .
$$

Thus the variation of refractive index due to the 3rd harmonic is proportional to the squared amplitude of the fundamental. $\gamma$ is the characteristic constant responsible for the $3 \mathrm{rd}$ order nonlinear polarization of the medium.

In the literature [22] the electrostriction of the medium is considered as the main reason of the nonlinear polarization and the coefficient $\gamma$ is expressed by the formula:

$$
\gamma=\frac{\rho\left(\frac{\partial \varepsilon}{\partial \rho}\right)_{\mathrm{s}} \beta_{\mathrm{s}}}{16 \pi n_{0}}
$$

where $\rho$ density, $\beta_{\mathrm{s}}$ adiabatic compressibility, $\varepsilon$ electric permeability, $n_{0}$ refractive index for undesturbed medium.

The quantities $\beta_{\mathrm{s}}$ and $(\rho \partial \varepsilon / \partial \rho)_{\mathrm{s}}$ are known for many substances [24] and it was possible to calculate $\Delta n$ from the formula (12) basing on (13) for chosen value of $E$. For the laser beam of power density $10^{12} \mathrm{~W} / \mathrm{m}^{2}$ what corresponds to

$$
E=2.7 \times 10^{7} \mathrm{~V} / \mathrm{m},
$$

$\gamma$ and $\Delta n$ were calculated for few liquids. Results are presented in the table $I$. 
TABLE I

\begin{tabular}{|c|c|c|c|c|c|c|}
\hline Substance & $n_{0}$ & $\begin{array}{l}\rho \times 10^{3} \\
{\left[\mathrm{~kg} / \mathrm{m}^{3}\right]}\end{array}$ & $\rho\left(\frac{\partial \varepsilon}{\partial \rho}\right)_{5}$ & $\begin{array}{c}\beta_{\mathrm{s}} \times 10^{13} \\
{\left[\mathrm{~N} / \mathrm{m}^{2}\right]}\end{array}$ & $\begin{array}{l}\gamma \times 10^{20} \\
{\left[\mathrm{~m}^{2} / \mathrm{V}^{2}\right]}\end{array}$ & $\Delta n \times 10^{6}$ \\
\hline 一 & 一 & - & - & - & - & 一 \\
\hline Water & $1.3397\left(^{c}\right)$ & $0.997\left(^{c}\right)$ & $0.82\left(^{c}\right)$ & $45.7\left(^{c}\right)$ & 0.06 & $\begin{array}{c}0.44 \\
{[0.38]\left({ }^{a}\right)}\end{array}$ \\
\hline Carbon disulfide & $1.674 \quad\left(^{a}\right)$ & $1.262\left(^{a}\right)$ & $2.39\left(^{a}\right)$ & $49.5\left(^{a}\right)$ & 0.42 & $\begin{array}{l}3.19 \\
{[2.91]\left({ }^{\alpha}\right)}\end{array}$ \\
\hline Benzene & $1.522\left(^{a}\right)$ & $0.879\left(^{a}\right)$ & $1.56\left(^{a}\right)$ & $52.6\left(^{a}\right)$ & 0.21 & $\begin{array}{l}1.61 \\
{[0.04]\left(^{\alpha}\right)}\end{array}$ \\
\hline Carbon tetrachloride & $1.4720\left({ }^{c}\right)$ & $1.595\left(^{c}\right)$ & $1.15\left(^{a}\right)$ & $58.0\left(^{c}\right)$ & 0.14 & 0.99 \\
\hline Toluene & $1.5130\left(^{c}\right)$ & $0.865\left(^{c}\right)$ & $1.60\left(^{c}\right)$ & $70.0\left(^{c}\right)$ & 0.30 & 2.24 \\
\hline Ethyl alcohol & $1.369 \quad\left({ }^{c}\right)$ & $0.789\left(^{c}\right)$ & $0.95\left(^{c}\right)$ & $92.8\left({ }^{c}\right)$ & 0.15 & 1.14 \\
\hline Methyl alcohol & $1.338 \quad\left({ }^{c}\right)$ & $0.791\left(^{c}\right)$ & $0.82\left(^{c}\right)$ & $100.5\left(^{c}\right)$ & 0.14 & 0.95 \\
\hline Nitrobenzene & $1.553\left(^{b}\right)$ & $1.203\left(^{a}\right)$ & $40.0 \quad\left(^{a}\right)$ & $34.8\left(^{a}\right)$ & 2.21 & 670.0 \\
\hline
\end{tabular}

$\left({ }^{a}\right)[22], \quad\left(^{b}\right)[23], \quad\left(^{c}\right)[24]$.

4. Light intensity distributions for diffraction patterns of the 3rd harmonic in some liquids. - In the case of the Raman-Nath's distribution there is no dependence on the kind of the liquid, so the distributions for the fundamental will be very similar in all liquids and look like shown in [20]. However, the distributions for the harmonic will be different in different liquids because its generation depends on the properties of the liquid.

From the formulas $\left(7^{\prime}\right)$ and with the values from the table $I$ the numerical calculations were carried out for different ultrasonic intensities (different values of $a$ which were the same as in [20] $\mathrm{i}$. e. $a=0.1-3$ ). Results of these calculations for the $3 \mathrm{rd}$ harmonic for nitrobenzene, carbon disulfide, benzene and carbon tetrachloride are presented in the figures 3 , 4,5 and 6 , respectively. In tense figures the relative values of the light intensity are drawn towards the value of $I_{0}^{0}$ which corresponds to the generated harmonics for the case when the ultrasonic wave is absent i. e. for $a=0$ and this value is equal to $100 \%$

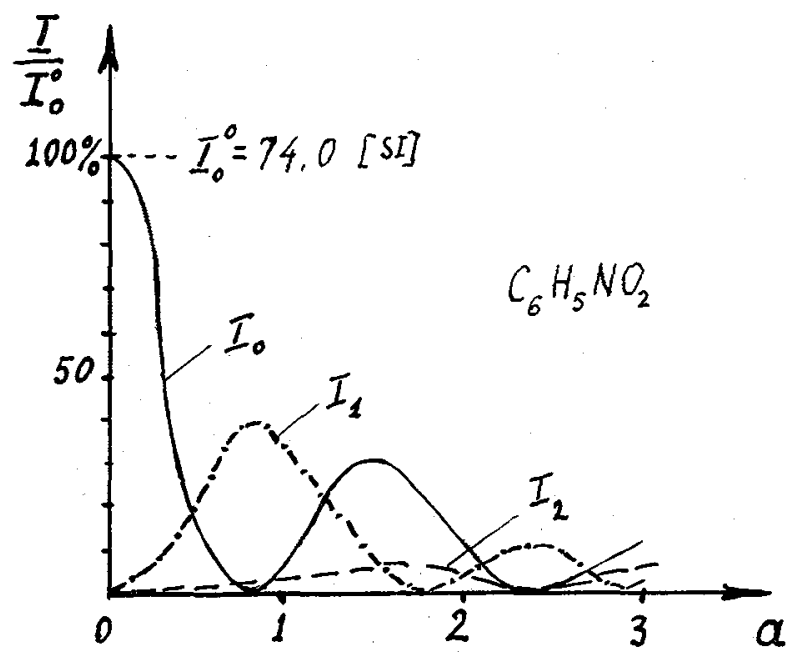

FIG. 3. (it corresponds to the value of 0.01 when $r=0.1$ was assumed in [20] for the case a)). The values of $I_{0}^{0}$ calculated from $\left(7^{\prime}\right)$ are marked at the ordinate axis.
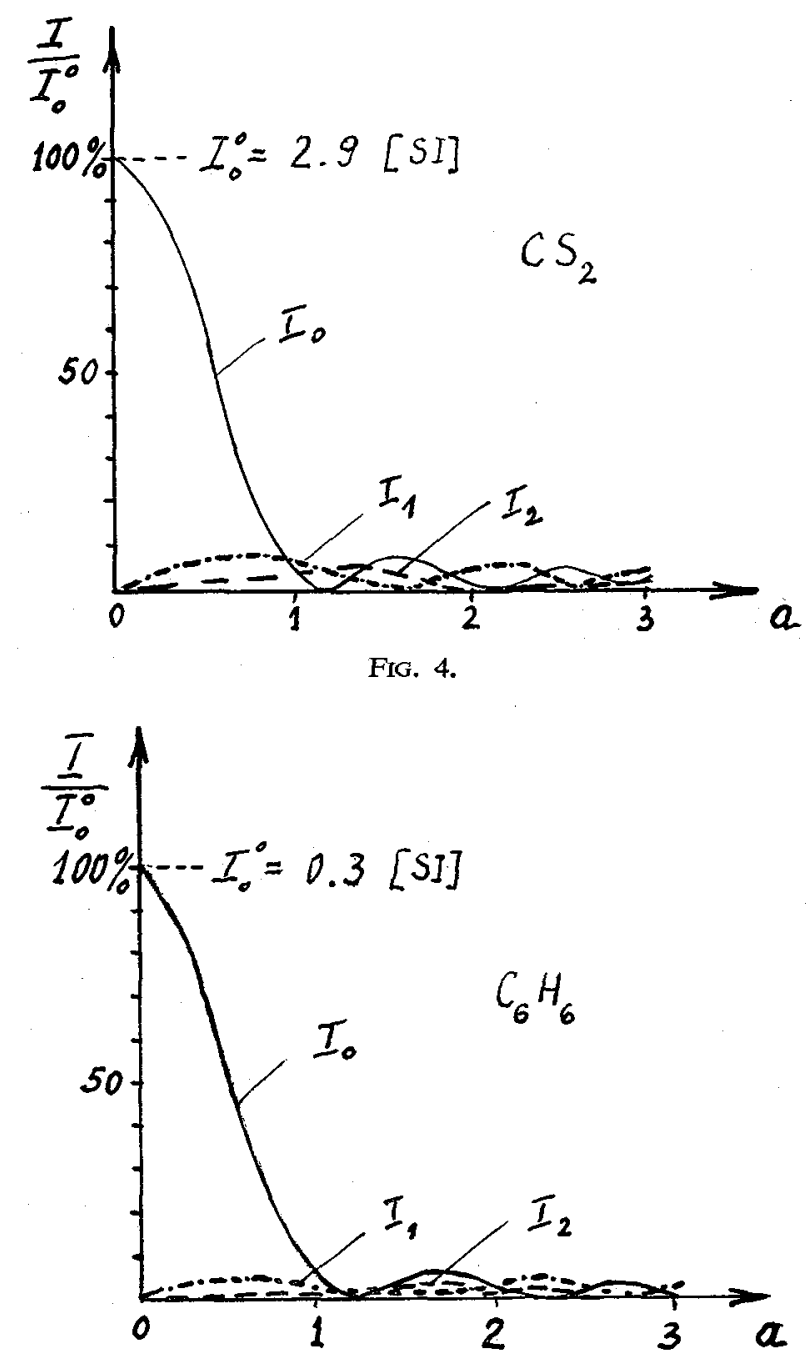

FiG. 5. 


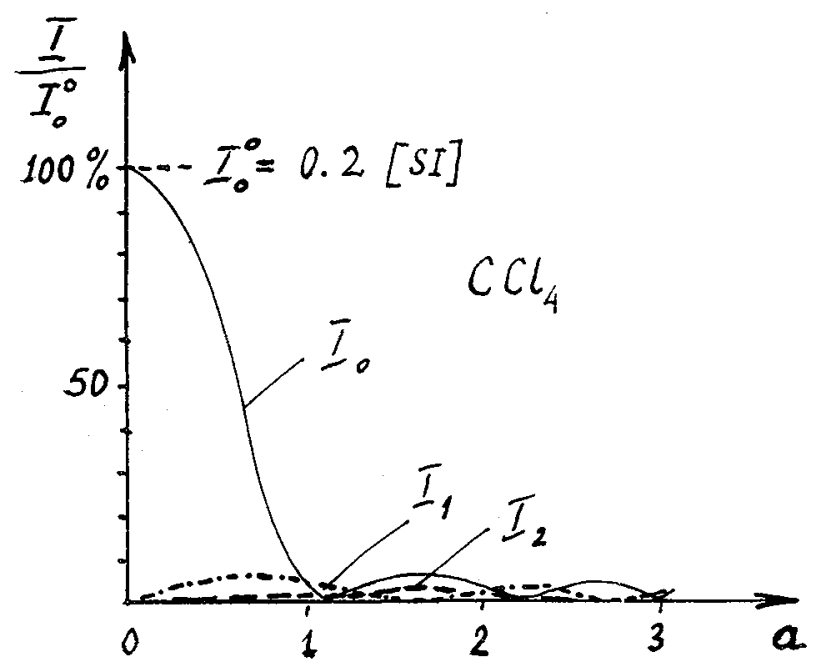

FIG. 6 .

It is seen that the distributions for relative intensities for different liquids are similar, however, the absolute values of the effect are strongly dependent on the substance what can be seen in the figure 7 where the contributions for different liquids to the 0 -order of diffraction (by the harmonic) $I_{0}$ is presented.

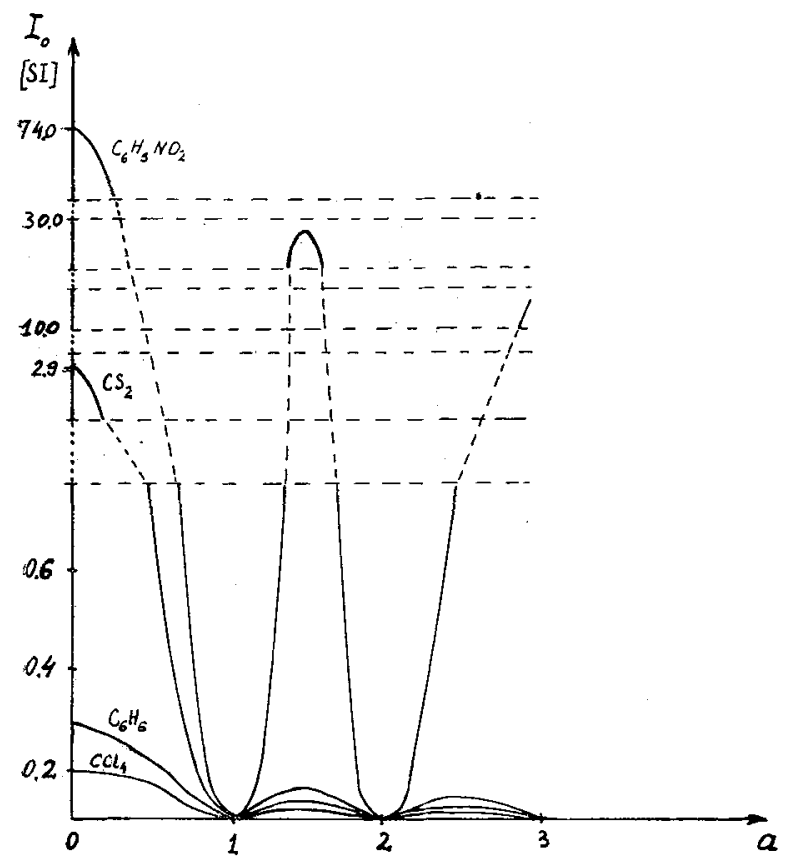

FIG, 7.

5. Some remarks on the experimental conditions for observing the phenomenon. - From the figure 7 as well as from the table I one can notice that the effect is large enough to be measured in such a liquid like nitrobenzene or carbon disulfide. Till now, so far as we know, nobody measured the generation of the 3 rd harmonic in these liquids.
There are only few papers where the generation of the 3rd harmonic of laser beam in liquids was measured : in some liquified organic scintillators [27] and in dye solutions [28].

They are mainly two practical difficulties to overcome to obtain generation of the 3rd harmonic [26]. One is to find the proper dependence of absorption against frequency. A suitable materialmust be relatively transparent to the fundamental and to the harmonic. The second is the matching in refractive indices of the fundamental and the harmonic what is closely connected with the coherence length expressed by [25], [27] :

$$
l_{\mathrm{s}}=\frac{\lambda_{\omega}}{6\left(n_{3 \omega}-n_{\omega}\right)},
$$

where $n_{3 \omega}$ and $n_{\omega}$ are refractive indices, $\lambda_{\omega}$ wavelength of fundamental. When the difference between indices is large then the coherence length is small.

The calculations of ultrasonic laser light diffraction are valid as we mentioned above for $l<l_{\mathrm{s}}$, so we must search for such a material where the thickness of the layer will be not too thin. For example in usual practical cases $l_{\mathrm{s}}$ calculated from last formula is the order of magnitude of few microns [27].

A promising results of 3rd harmonic generation in the layer of $1 \mathrm{~mm}$ were obtained in [28] in spite of dismatching of refractive indices only for convenient relations of absorption.

Regarding to the liquids considered above nitrobenzene presents rather a convenient substance from the absorption point of view, it is transparent for ruby laser light as for the fundamental $(80 \%)$ (6 $943 \AA$ ) as for the 3rd harmonic (75\%) (2 $314 \AA)$ [29]. Unfortunately, the index matching problem is difficult to estimate because the index of nitrobenzene in the vicinity of $2300 \AA$ is not exactly known. Only from very rough evaluation one can say that the coherent length will not be greater than some scores of microns.

Thus, in our experiments with the ruby laser which we have started with we cannot follow a beaten track and as yet, we are not able to present any positive experimental data.

6. Conclusions. - The approximate theory of ultrasonic laser light diffraction discussed in this paper and based on the Raman-Nath's approach, gives a possibility to evaluate a magnitude of the influence of 3rd harmonic on the intensity distribution in diffraction pattern. The effect depends on the electrostrictive properties of a medium. Generally, it is rather small but in some organic liquids large enough to be measured. Experimental difficulties are connected with the generation and detection of the 3rd harmonic in liquids itself and with the interaction of it with ultrasound within the coherence length. 


\section{References}

[1] Lucas R., BiQuard P., C. R. Acad. Sci., 194 (1932), 2132 ; J. Physique Rad., 3 (1932), 464.

[2] Debye P., Sears F. W., Proc. Nat. Acad. Sci. Wash., 18 (1932), 409.

[3] Brillouin L., Annls de Phys. (9), 17 (1922), 88.

[4] Lucas R., Biquard P., J. Physique Rad., 5 (1934), 119.

[5] Raman C. V., Nagendra-Nath N. S., Proc. Ind. Acad. Sci., A 2 (1935), 406 ; A 2 (1935), 413 ; A 3 (1936), 75 ; A 3 (1936), $119 ; A 3$ (1936), 459.

[6] Wannier G., Extermann R., Helv. Phys. Acta, 9 (1936), $337 ; 9$ (1936), 520.

[7] Rytow S. M., Izw. Ak. Nauk SSSR, (1937), 233.

[8] Wagner H., Z. Phys., 141 (1955), 604, 622.

[9] Bathia A. B., Noble W. J., Proc. R. Soc., A 220 (1953), 356.

[10] David E., Phys. Z., 38 (1937), 587 ; 38 (1937), 592.

[11] Mertens R., Z. Phys., 160 (1960), 291.

[12] BERRY M. V., The diffraction of light by ultrasound, Academic Press, London-New York (1966).

[13] Born M., Wolf E., Principles of Optics, Pergamon Press, Oxford (1964).

[14] Mikhaylov I. G., Shutmov V. A., Akust. Zhurn., 3 (1957), $203 ; 4$ (1958), 174 ; 5 (1959), 77.
[15] Zankel K. L., Hiedemann E. A., J. Acoust. Soc. Am., 31 (1959), 44.

[16] Murthy J. S., J. Acoust. Soc. Am., 26 (1954), 970.

[17] Phariseau P., Physica, 25 (1959), 917.

[18] Sliwinski A., Proc. of 3rd Conf. on Acoust. Budapest (1964), 392.

[19] Sluwinski A., Arch. Akust., 1 (1970), 85.

[20] Jozefowska J., Sliwinski A., Proc 7th Int. Congr. Acoust. Budapest (1971), 21 U 5.

[21] Proc. of Conf. on Ultrasound, Prague (1972).

[22] Kaczmarek F., Zagadnienia Fizyki Dielektryków, Probl. Elektr. i Telekom. 22 (1970).

[23] Piekara A., Nowe oblicze optyki, PWN, Warszawa (1968).

[24] Fabielinskij I. L., Molekularnoye, rassijanije svieta, Moskva (1965).

[25] Kielich S., Post, Fizyki, 22 (1971), 24.

[26] Franken P. A., Ward J. F., Rev. Mod. Phys., 35 (1963), 23.

[27] Topp M. R., Jones R. P., Rentzepis P. M., Opt. Commun, 3 (1971), 264.

[28] Bey P. P., Guiliani J. F., Rabin H., Phys. Rev. Lett., 19 (1967), 819.

[29] Silverstein R. M., Bassler G. C., Spectrometric Identification of Organic Compounds, J. Wiley \& Sons, N. York-London-Sydney (1967). 